MATHEMATICS OF COMPUTATION

S 0025-5718(99)01089-3

Article electronically published on February 10, 1999

\title{
CONVERGENCE OF RELAXATION SCHEMES FOR HYPERBOLIC CONSERVATION LAWS WITH STIFF SOURCE TERMS
}

\author{
A. CHALABI
}

\begin{abstract}
We focus in this study on the convergence of a class of relaxation numerical schemes for hyperbolic scalar conservation laws including stiff source terms. Following Jin and Xin, we use as approximation of the scalar conservation law, a semi-linear hyperbolic system with a second stiff source term. This allows us to avoid the use of a Riemann solver in the construction of the numerical schemes. The convergence of the approximate solution toward a weak solution is established in the cases of first and second order accurate MUSCL relaxed methods.
\end{abstract}

\section{INTRODUCTION}

Hyperbolic conservation laws with stiff source terms could describe the effect of relaxation as in the kinetic theory of gases, elasticity with memory, water waves, traffic flows, etc.

These problems may be mathematically written in scalar case as a Cauchy problem of the form

$$
\begin{gathered}
\left.u_{t}+f(u)_{x}=q(u), \quad(x, t) \in \mathbb{R} \times\right] 0, T[; T>0 \\
u(x, 0)=u_{0}(x), \quad x \in \mathbb{R} .
\end{gathered}
$$

Theoretical study of the relaxation phenomena may be found in Chen et al. [7], Liu [20], and Whitham [32].

Numerical methods have been derived for the approximation of the conservation laws including nonstiff source terms in [3], [4], [24], [26], and [29]. These methods are based on explicit difference schemes. It is well known that explicit schemes are not appropriate for the numerical treatment of the stiff source terms; this motivates the use of semi-implicit and fully implicit schemes.

The approximation of the stiff case was recently studied by several authors (see [2], [6], [8], [13], [15], [17], [19], [25], and [26]), where different methods like asymptotic or splitting methods are used.

The main difficulty when we deal with the numerical solution of the stiff problems is the wrong location of the discontinuities. This problem has been investigated in [2], [8], [11], [13], [15], [17], and [19].

Received by the editor April 29, 1997 and, in revised form, October 14, 1997.

1991 Mathematics Subject Classification. Primary 35L65, 65M05, 65M10.

Key words and phrases. Conservation laws, stiff source term, relaxation scheme, fully implicit scheme, semi-implicit scheme, TVD scheme, MUSCL method, entropy solution. 
Error bounds related to the approximation of (1.1)-(1.2) were derived in [5], [25] and $[29]$.

In a recent study, Jin and Xin ([16]) introduced explicit relaxing schemes for the approximation of systems of hyperbolic conservation laws without source terms. Our purpose in this paper is the study of the convergence of semi-implicit relaxed schemes for the approximation of scalar conservation laws, including stiff source terms.

We do not assume here that the source term $q$ is a Lipschitzian function in $u$ but we suppose that $q^{\prime} \leq 0$. This last hypothesis is realistic since it does indicate the dissipativity of the source term $q$ in the sense of [7]. This is the case in the models of combustion ([2], [11], [21]), gas dynamics with heat transfer ([13]), water waves in the presence of the friction force of the bottom ([7]), etc. We point out that in all these examples and many others the source term is not a Lipschitzian function. The nonpositivity of $q^{\prime}$ is also assumed by Liu in [20] and Chen et al. in [7].

Thanks to the implicit character and to the dissipativity of the source term $q$ (nonpositivity of $q^{\prime}$ ), some proposed schemes are TVD, entropy satisfying at the limit, and they are monotone in the first order accurate semi-implicit case. Then they possess almost all the same properties as in the homogeneous schemes $(q=0)$.

This paper is organized as follows. Section 2 is devoted to recalling some preliminaries related to the hyperbolic conservation laws, including source terms. At first we give results which summarize the properties of the exact solution of the Cauchy problem when $q^{\prime}$ is nonpositive. These properties are similar to those related to the homogeneous case. In Section 3, we give an error bound for the relaxation problem. Section 4 concerns the upwind semi-implicit relaxing schemes. Section 5 is devoted to the study of the first and second order accurate relaxed schemes. An extension to the two dimensional case is presented in Section 6. Finally, in Section 7, we give concluding remarks.

\section{Preliminaries}

We seek a weak solution to the Cauchy problem (1.1)-(1.2); that is a function $u \in L^{\infty}(\mathbb{R} \times] 0, T[)$ satisfying

$$
\int_{\mathbb{R}} \int_{] 0, T[}\left[u \varphi_{t}+f(u) \varphi_{x}\right] d x d t+\int_{\mathbb{R}} u_{0}(x) \varphi(x, 0) d x=-\int_{\mathbb{R}} \int_{] 0, T[} q(u) \varphi(x, t) d x d t
$$

for all $\varphi \in \mathcal{C}^{1}(\mathbb{R} \times[0, T[)$, with compact support in $(\mathbb{R} \times[0, T[)$.

Let $\eta \in \mathcal{C}^{2}(\mathbb{R})$ be a strictly convex function, whose entropy flux is $F$, that is

$$
\eta^{\prime}(u) f^{\prime}(u)=F^{\prime}(u) \quad \forall u \in \mathbb{R} .
$$

The solution of (1.1)-(1.2) is not necessarily unique and the physical one is characterized by the following entropy condition:

$$
\begin{gathered}
\int_{\mathbb{R}} \int_{] 0, T[}\left[\eta(u) \varphi_{t}+F(u) \varphi_{x}\right] d x d t+\int_{\mathbb{R}} \eta\left(u_{0}(x)\right) \varphi(x, 0) d x \\
\geq-\int_{\mathbb{R}} \int_{] 0, T[} \eta^{\prime}(u) q(u) \varphi(x, t) d x d t
\end{gathered}
$$

for all test positive functions $\varphi \in \mathcal{C}^{1}(\mathbb{R} \times[0, T[)$, with compact support in $\mathbb{R} \times[0, T$. 
Let $u(x, t)=S(t) u_{0}$ denote the unique weak solution of (1.1)-(1.2) which satisfies the entropy condition. Let us assume that

$$
\sup _{u} q^{\prime}(u) \leq \gamma, \quad \gamma=\text { constant. }
$$

Using a result of Kruzkov ([18]), we can easily prove the following

Proposition 2.1. If $u_{0} \in B V(\mathbb{R}) \cap L^{1}(\mathbb{R}), f \in \mathcal{C}^{1}(\mathbb{R}), q \in \mathcal{C}^{1}(\mathbb{R})$ such that $q(0)=$ 0 and $q^{\prime} \leq 0$, then the problem (1.1)-(1.2) possesses a unique entropy solution $u(x, t)=S(t) u_{0}$ satisfying:

i) $\left\|S(t) u_{0}\right\|_{L^{\infty(\mathbb{R})} \leq \|} \leq u_{0} \|_{L^{\infty}(\mathbb{R})}$;

ii) Moreover, for any $v_{0} \in L^{\infty}(\mathbb{R})$ we have

$$
\left\|S(t) u_{0}-S(t) v_{0}\right\|_{L^{1}(\mathbb{R})} \leq\left\|u_{0}-v_{0}\right\|_{L^{1}(\mathbb{R})} ;
$$

iii) $T V\left(S(t) u_{0}\right) \leq T V\left(u_{0}\right)$;

iv) If $u_{0}(x) \leq v_{0}(x)$, then $S(t) u_{0} \leq S(t) v_{0}$.

Remark 2.1. If we suppose that $q \in \mathcal{C}^{1}(\mathbb{R})$ such that $q(0)=0$ and $q^{\prime} \leq 0$, then $q$ is dissipative in the sense of Chen et al. ([7]). Indeed if we take $\eta(u)=u^{2} / 2$ as an entropy function and apply the mean value theorem to $q$, we get

$$
\begin{aligned}
\eta^{\prime}(u) q(u) & =u\left[q(0)+u q^{\prime}(\xi)\right] \quad(\min (0, u)<\xi<\max (0, u)) \\
& =q^{\prime}(\xi) u^{2} \leq 0 .
\end{aligned}
$$

Let $h$ be the spatial size and $k$ be the time grid size related to $h$ by the fixed positive number $r$ through

$$
r=k / h \text {. }
$$

A weak solution of (2.1)-(2.3) is approximated by a function $u_{h}$ defined on $\mathbb{R} \times] 0, T[$ by

$$
u_{h}(x, t)=u_{j}^{n} \text { for }(x, t) \in I_{j} \times J_{n}
$$

with

$$
\left.I_{j} \times J_{n}=\right](j-1 / 2) h,(j+1 / 2) h[\times](n-1 / 2) k,(n+1 / 2) k[
$$

for all $j \in \mathbb{Z}$ and $n \leq N=E(T / k)+1$, where $E$ denotes the integer part function.

The initial condition (2.2) is projected into the space of piecewise constant functions by

$$
u_{j}^{0}=\frac{1}{h} \int_{I_{j}} u_{0}(x) d x \quad \forall j \in \mathbb{Z} .
$$

In all the following, we assume that

$$
\begin{gathered}
u_{0} \in B V(\mathbb{R}), \quad f \in \mathcal{C}^{1}(\mathbb{R}), \\
q \in \mathcal{C}^{1}(\mathbb{R}) \quad \text { such that } \quad q(0)=0, \quad q^{\prime} \leq 0 .
\end{gathered}
$$




\section{The RELAXATION PROBLEM}

Now, as in [16], let us associate with the scalar Cauchy problem (1.1)-(1.2), the semi-linear relaxation system

$$
\left\{\begin{array}{l}
u_{t}+v_{x}=q(u) \\
v_{t}+a u_{x}=-\frac{1}{\epsilon}(v-f(u))
\end{array}\right.
$$

with the initial data

$$
u(x, 0)=u_{0}(x), \quad v(x, 0)=v_{0}(x)=f\left(u_{0}(x)\right),
$$

where the small parameter $\epsilon$ is the relaxation rate and $a$ is a positive constant. Let $\left(u_{\epsilon}, v_{\epsilon}\right)$ be the solution of the system (3.1). If we consider a formal expansion of Hilbert type, we get

$$
v^{\epsilon}=f\left(u^{\epsilon}\right)+\epsilon v_{1}^{\epsilon}+\epsilon^{2} v_{2}^{\epsilon}+\cdots .
$$

We obtain as a first order approximation of the system (3.1)

$$
u_{t}+f(u)_{x}=q(u)+\epsilon\left(f^{\prime}(u) q(u)\right)_{x}+\epsilon\left(\left(a-f^{\prime}(u)^{2}\right) u_{x}\right)_{x} .
$$

Thus, (3.3) is dissipative if the following subcharactrestic condition is satisfied:

$$
-\sqrt{a} \leq f^{\prime}(u) \leq \sqrt{a} \quad \text { for all } u .
$$

Let $Q=\mathbb{R} \times] 0, T$, then by similar proof to that in [9], we prove

Theorem 3.1. Suppose that the initial data is bounded and the subcharacteristic condition (3.4) is satisfied, then

i) The relaxation problem (3.1)-(3.2) admits a unique solution.

ii) If we suppose further that there is no interval on which the flux function $f$ is affine, then the solution $\left(u^{\epsilon}, v^{\epsilon}\right)$ of $(3.1)-(3.2)$ converges in $L^{p}(Q) \times$ $L^{2}(Q), 0<p<\infty$ toward $(u, f(u))$ as $\epsilon$ tends to zero, where $u$ is the entropy solution of (1.1)-(1.2).

iii) We have the bound error:

$$
\left\|f\left(u^{\epsilon}\right)-v^{\epsilon}\right\|_{L^{2}(Q)} \leq C \sqrt{\epsilon}
$$

where $C$ is a positive constant.

Proof. The proof of i) is based on invariant regions (see [9]). Using the entropy inequality we obtain ii).

Let us prove property iii) as we do in the homogeneous case in [14]. Let $(\eta(u, v), F(u, v))$ be a convex entropy-flux entropy function associated with the system (3.1) such that

$$
a \eta_{v}=F_{u}, \quad \eta_{u}=F_{v}
$$

by multiplying the first equation of (3.1) by $\eta_{u}$ and the second equation of (3.1) by $\eta_{v}$. Summing the obtained equations, we get

$$
\frac{\partial}{\partial t} \eta(u, v)+\frac{\partial}{\partial x} F(u, v)=\frac{1}{\epsilon} \eta_{v}(f(u)-v)+q(u) \eta_{u} .
$$


Hence,

$$
\begin{aligned}
& \frac{1}{\epsilon} \int_{Q} \eta_{v}(v-f(u)) d x d t-\int_{Q} q(u) \eta_{u} d x d t \\
& \quad=\int_{\mathbb{R}} \eta(u(x, T), v(x, T)) d x-\int_{\mathbb{R}} \eta\left(u_{0}(x), v_{0}(x)\right) d x .
\end{aligned}
$$

Thus

$$
\frac{1}{\epsilon} \int_{Q} \eta_{v}(v-f(u)) d x d t-\int_{Q} q^{\prime}(\xi) u^{2} \eta_{u u}(\alpha, \beta) \leq \int_{\mathbb{R}} \eta\left(u_{0}(x), v_{0}(x)\right) d x d t .
$$

We have

$$
\eta_{v}(u, v)=(v-f(u)) \eta_{v v}(\lambda, \nu) .
$$

Using the convexity and the nonpositivity of $q^{\prime}$, we get

$$
\frac{1}{\epsilon} \int_{Q} \eta_{v v}(\lambda, \nu)(v-f(u))^{2} d x d t \leq \int_{\mathbb{R}} \eta\left(u_{0}(x), v_{0}(x)\right) d x d t .
$$

Then

$$
\left\|f\left(u^{\epsilon}\right)-v^{\epsilon}\right\|_{L^{2}(Q)} \leq C \sqrt{\epsilon}
$$

\section{The UPWIND SEMI-IMPLICIT RELAXING SCHEME}

The linear hyperbolic part of the system (3.1) has two Riemann invariants

$$
v+\sqrt{a} u \text { and } v-\sqrt{a} u
$$

associated with the characteristic fields $\lambda_{-}=-\sqrt{a}$ and $\lambda_{+}=+\sqrt{a}$.

The first-order upwind scheme applied to $v \pm \sqrt{a} u$ gives

$$
(v+\sqrt{a} u)_{j+1 / 2}=(v+\sqrt{a} u)_{j}, \quad(v-\sqrt{a} u)_{j+1 / 2}=(v-\sqrt{a} u)_{j+1} ;
$$

hence,

$$
\begin{aligned}
& u_{j+1 / 2}=\frac{1}{2}\left(u_{j}+u_{j+1}\right)-\frac{1}{2 \sqrt{a}}\left(v_{j+1}-v_{j}\right), \\
& v_{j+1 / 2}=\frac{1}{2}\left(v_{j}+v_{j+1}\right)-\frac{1}{2} \sqrt{a}\left(u_{j+1}-u_{j}\right) .
\end{aligned}
$$

We can then construct the following upwind scheme:

$$
\left\{\begin{array}{l}
\frac{1}{k}\left(u_{j}^{n+1}-u_{j}^{n}\right)+\frac{1}{2 h}\left(v_{j+1}^{n}-v_{j-1}^{n}\right)-\frac{\sqrt{a}}{2 h}\left(u_{j+1}^{n}-2 u_{j}^{n}+u_{j-1}^{n}\right)=q\left(u_{j}^{n+1}\right) \\
\frac{1}{k}\left(v_{j}^{n+1}-v_{j}^{n}\right)+\frac{a}{2 h}\left(u_{j+1}^{n}-u_{j-1}^{n}\right)-\frac{\sqrt{a}}{2 h}\left(v_{j+1}^{n}-2 v_{j}^{n}+v_{j-1}^{n}\right) \\
\quad=-\frac{1}{\epsilon}\left(v_{j}^{n+1}-f\left(u_{j}^{n+1}\right)\right) .
\end{array}\right.
$$

For the existence and uniqueness of the solution of (4.1), we prove the following result.

Proposition 4.1. The system (4.1) admits a unique solution $\left(u_{j}^{n+1}, v_{j}^{n+1}\right), n \in$ $\mathbb{N}, j \in \mathbb{Z}$. 
Proof. It suffices to prove that the first equation of (4.1) admits a unique solution $\left(u_{j}^{n+1}\right)$. Indeed the function

$$
x \longmapsto x-k q(x)
$$

is differentiable and strictly monotone since $q^{\prime} \leq 0$. Then the first equation of (4.1) admits a unique solution $u_{j}^{n+1}$.

\section{THE RELAXED SCHEMES}

By making use of the Hilbert expansion again, applied to (4.1), we get as $\epsilon$ tends to zero, the following relaxed schemes.

5.1. The upwind semi-implicit relaxed scheme. Using a formal expansion of Hilbert type in (4.1), we get the following relaxed scheme:

$$
\left\{\begin{array}{l}
v_{j}^{n}=f\left(u_{j}^{n}\right) \\
u_{j}^{n+1}=u_{j}^{n}-\frac{r}{2}\left(f\left(u_{j+1}^{n}\right)-f\left(u_{j-1}^{n}\right)\right)+\frac{\sqrt{a} r}{2}\left(u_{j+1}^{n}-2 u_{j}^{n}+u_{j-1}^{n}\right)+k q\left(u_{j}^{n+1}\right) .
\end{array}\right.
$$

To deal with locally bounded variation functions, we set

$$
\begin{aligned}
\Omega_{R} & =]-R, R[\times] 0, T[, \quad \text { for } R>0, \\
J & =E(R / h), \quad \text { where } E \text { is the integer part function. }
\end{aligned}
$$

Throughout this section, we set

$$
L=\sup _{u \in \mathcal{A}}\left|f^{\prime}(u)\right|, \quad M=\sup _{u \in \mathcal{A}}\left|q^{\prime}(u)\right|,
$$

where

$$
\mathcal{A}=\left\{v \in L^{\infty}(\mathbb{R}),\|v\|_{L^{\infty}(\mathbb{R})} \leq\left\|u_{0}\right\|_{L^{\infty}(\mathbb{R})}\right\} .
$$

Proposition 5.1. Under the subcharacteristic condition (3.4) and the CFL condition

$$
r \sqrt{a} \leq 1
$$

we have

i) The scheme (5.1) is monotone.

ii) $\left\|u^{n+1}\right\|_{L^{\infty}(\mathbb{Z})} \leq\left\|u^{n}\right\|_{L^{\infty}(\mathbb{Z})}$.

iii) $T V\left(u^{n+1}\right) \leq T V\left(u^{n}\right)$; that is, the scheme (5.1) is TVD.

iv) $\sum_{|j| \leq J}\left|u_{j}^{n+1}-u_{j}^{n}\right| \leq C_{1} T V\left(u^{n}\right)+C_{2}\left\|u^{n+1}\right\|_{L^{\infty}(\mathbb{Z})}, C_{1}, C_{2}$ are positive constants.

Proof. First we write the scheme (5.1) in the form

$$
\left\{\begin{array}{l}
u_{j}^{n+1}=\bar{u}_{j}^{n+1}+k q\left(u_{j}^{n+1}\right) \\
\bar{u}_{j}^{n+1}=u_{j}^{n}-\frac{r}{2}\left(f\left(u_{j+1}^{n}\right)-f\left(u_{j-1}^{n}\right)\right)+\frac{\sqrt{a} r}{2}\left(u_{j+1}^{n}-2 u_{j}^{n}+u_{j-1}^{n}\right) .
\end{array}\right.
$$

i) To show that the scheme (5.1) is monotone, let us suppose that

$$
u_{j}^{n} \leq w_{j}^{n} \quad \forall j \in \mathbb{Z} .
$$

Using (5.3), we have

$$
\left[u_{j}^{n+1}-w_{j}^{n+1}\right]\left[1-k q^{\prime}\left(\alpha_{j}^{n}\right)\right]=\bar{u}_{j}^{n+1}-\bar{w}_{j}^{n+1} .
$$


The second equation of (5.3) is a monotone scheme under the subcharacteristic condition (3.4) and the CFL condition (5.2), then

$$
u^{n} \leq w^{n} \Rightarrow \bar{u}^{n+1} \leq \bar{w}^{n+1} .
$$

Using the nonpositivity of $q^{\prime}$, we obtain

$$
u_{j}^{n+1} \leq w_{j}^{n+1} \quad \forall j \in \mathbb{Z} .
$$

ii) Using (5.3), we have

$$
u_{j}^{n+1}-k q\left(u_{j}^{n+1}\right)=\bar{u}_{j}^{n+1} .
$$

Thus

$$
\left(1-k q^{\prime}\left(\xi_{j}^{n}\right)\right) u_{j}^{n+1}=\bar{u}_{j}^{n+1},
$$

where $q^{\prime}$ is nonpositive, then we obtain

$$
\left\|u^{n+1}\right\|_{L^{\infty}(\mathbb{Z})} \leq\left\|\bar{u}^{n+1}\right\|_{L^{\infty}(\mathbb{Z})} .
$$

Taking into account the subcharacteristic condition (3.4) and the CFL condition (5.2), we easily show that

$$
\left\|\bar{u}^{n+1}\right\|_{L^{\infty}(\mathbb{Z})} \leq\left\|u^{n}\right\|_{L^{\infty}(\mathbb{Z})} .
$$

Then

$$
\left\|u^{n+1}\right\|_{L^{\infty}(\mathbb{Z})} \leq\left\|u^{n}\right\|_{L^{\infty}(\mathbb{Z})} .
$$

iii) By making use of (5.3), we get

$$
\left[u_{j}^{n+1}-u_{j-1}^{n+1}\right]\left[1-k q^{\prime}\left(\alpha_{j}^{n}\right)\right]=\bar{u}_{j}^{n+1}-\bar{u}_{j-1}^{n+1},
$$

and the nonpositivity of $q^{\prime}$ gives us

$$
T V\left(u^{n+1}\right) \leq T V\left(u^{n}\right) .
$$

iv) From (5.1), we obtain

$$
\begin{aligned}
u_{j}^{n+1}-u_{j}^{n}= & -\frac{r}{2}\left[\left(f\left(u_{j+1}^{n}\right)-f\left(u_{j}^{n}\right)\right)+\left(f\left(u_{j}^{n}\right)-f\left(u_{j-1}^{n}\right)\right)\right] \\
& +\frac{\sqrt{a} r}{2}\left[\left(u_{j+1}^{n}-u_{j}^{n}\right)-\left(u_{j}^{n}-u_{j-1}^{n}\right)\right]+k q\left(u_{j}^{n+1}\right) .
\end{aligned}
$$

Hence,

$$
\begin{aligned}
\sum_{|j| \leq J} \mid u_{j}^{n+1}- & u_{j}^{n} \mid \leq \frac{r}{2} L\left(\sum_{|j| \leq J}\left|u_{j+1}^{n}-u_{j}^{n}\right|+\sum_{|j| \leq J}\left|u_{j}^{n}-u_{j-1}^{n}\right|\right) \\
& +\frac{\sqrt{a} r}{2}\left(\sum_{|j| \leq J}\left|u_{j+1}^{n}-u_{j}^{n}\right|+\sum_{|j| \leq J}\left|u_{j}^{n}-u_{j-1}^{n}\right|\right)+k M \sum_{|j| \leq J}\left|u_{j}^{n+1}\right| .
\end{aligned}
$$

Then

$$
\sum_{|j| \leq J}\left|u_{j}^{n+1}-u_{j}^{n}\right| \leq C_{1} T V\left(u^{n}\right)+C_{2}\left\|u^{n+1}\right\|_{L^{\infty}(\mathbb{Z})}
$$

with

$$
C_{1}=r(L+\sqrt{a}), \quad C_{2}=r R M \quad \text { since } \quad J h \leq R .
$$

Theorem 5.1. Under the subcharacteristic condition (3.4) and the CFL condition (5.2), the approximate solution $u_{h}$ constructed by the semi-implicit scheme (5.1) converges in $L_{\mathrm{loc}}^{1}(\mathbb{R} \times] 0, T[)$ toward the entropy satisfying solution of (1.1)-(1.2), as $h$ tends to zero. 
Proof. Using Proposition 5.1, we obtain that the sequence $\left(u_{h}\right)$ is bounded in family in the space $L^{\infty}(\mathbb{R} \times] 0, T[) \cap B V_{\text {loc }}(\mathbb{R} \times] 0, T[)$. By a similar proof as in [6], we make use of Helly's theorem to get a subsequence extracted from $\left(u_{h}\right)$ which converges toward the entropy solution of (1.1)-(1.2), and the uniqueness of the entropy satisfying solution ensures that all the sequence $\left(u_{h}\right)$ converges toward the entropy satisfying solution $u$ of problem (1.1)-(1.2) as $h$ tends toward zero.

5.2. The upwind fully implicit relaxed scheme. Here, we consider the following implicit relaxed scheme:

$\left\{\begin{array}{l}v_{j}^{n}=f\left(u_{j}^{n}\right) \\ u_{j}^{n+1}=u_{j}^{n}-\frac{r}{2}\left(f\left(u_{j+1}^{n+1}\right)-f\left(u_{j-1}^{n+1}\right)\right)+\frac{\sqrt{a} r}{2}\left(u_{j+1}^{n+1}-2 u_{j}^{n+1}+u_{j-1}^{n+1}\right)+k q\left(u_{j}^{n+1}\right) .\end{array}\right.$

First, we introduce, as in [31], the operator $T_{\mu}$ defined on piecewise constant functions by

$$
T_{\mu}\left(u_{h}\right)_{j}=u_{j}-\frac{\mu r}{2}\left(f\left(u_{j+1}\right)-f\left(u_{j-1}\right)\right)+\frac{\sqrt{a} \mu r}{2}\left(u_{j+1}-2 u_{j}+u_{j-1}\right), \quad j \in \mathbb{Z},
$$

where $\mu$ is a parameter satisfying $\mu>0$, and $u_{h}=\left(u_{j}\right)_{j \in \mathbb{Z}}$.

By a similar proof to that in [31] we can prove

Lemma 5.1. Let $u_{h}=\left(u_{j}\right)_{j \in \mathbb{Z}}$ be a piecewise constant function. If the subcharacteristic condition (3.4) is satisfied and if the positive parameter $\mu$ is sufficiently small such that

$$
\mu r \sqrt{a} \leq 1
$$

then

i) $\left\|T_{\mu}\left(u_{h}\right)\right\|_{L^{\infty}(\mathbb{Z})} \leq\left\|u_{h}\right\|_{L^{\infty}(\mathbb{Z})}$;

ii) $T V\left(T_{\mu}\left(u_{h}\right)\right) \leq T V\left(u_{h}\right)$;

iii) $\left\|T_{\mu}\left(u_{h}\right)-T_{\mu}\left(v_{h}\right)\right\|_{L^{1}(\mathbb{Z})} \leq\left\|u_{h}-v_{h}\right\|_{L^{1}(\mathbb{Z})}$.

Next, we prove

Proposition 5.2. Under the subcharacteristic condition (3.4), the approximate solution given by (5.4) satisfies the following properties:

i) $\left\|u^{n+1}\right\|_{L^{\infty}(\mathbb{Z})} \leq\left\|u^{n}\right\|_{L^{\infty}(\mathbb{Z})}$;

ii) $T V\left(u^{n+1}\right) \leq T V\left(u^{n}\right)$ (TVD character);

iii) $\sum_{|j| \leq J}\left|u_{j}^{n+1}-u_{j}^{n}\right| \leq C_{1} T V\left(u^{n+1}\right)+C_{2}\left\|u^{n+1}\right\|_{L^{\infty}(\mathbb{Z})}$.

Proof. i) Using the operator $T_{\mu}$ defined in (5.5), the scheme (5.4) may be written

$$
u^{n+1}=\frac{\mu}{1+\mu} u^{n}+\frac{1}{1+\mu} T_{\mu}\left(u^{n+1}\right)+\frac{\mu}{1+\mu} k q\left(u^{n+1}\right) .
$$

Applying the mean value theorem to $q$, we get

$$
\left[1-\frac{\mu}{1+\mu} k q^{\prime}\left(\xi^{n+1}\right)\right] u^{n+1}=\frac{\mu}{1+\mu} u^{n}+\frac{1}{1+\mu} T_{\mu}\left(u^{n+1}\right) .
$$

Taking into account the nonpositivity of $q^{\prime}$, we obtain

$$
\left\|u^{n+1}\right\|_{L^{\infty}(\mathbb{Z})} \leq \frac{\mu}{1+\mu}\left\|u^{n}\right\|_{L^{\infty}(\mathbb{Z})}+\frac{1}{1+\mu}\left\|T_{\mu}\left(u^{n+1}\right)\right\|_{L^{\infty}(\mathbb{Z})} .
$$


By making use of Lemma 5.1, we have

$$
\left\|T_{\mu}\left(u^{n+1}\right)\right\|_{L^{\infty}(\mathbb{Z})} \leq\left\|u^{n+1}\right\|_{L^{\infty}(\mathbb{Z})} .
$$

Hence,

$$
\left\|u^{n+1}\right\|_{L^{\infty}(\mathbb{Z})} \leq \frac{\mu}{1+\mu}\left\|u^{n}\right\|_{L^{\infty}(\mathbb{Z})}+\frac{1}{1+\mu}\left\|u^{n+1}\right\|_{L^{\infty}(\mathbb{Z})} .
$$

Then

$$
\left\|u^{n+1}\right\|_{L^{\infty}(\mathbb{Z})} \leq\left\|u^{n}\right\|_{L^{\infty}(\mathbb{Z})} .
$$

The properties ii) and iii) may be proved as in the former semi-explicit case.

Proposition 5.3. If the subcharacteristic condition (3.4) is satisfied and

$$
k \sup _{u \in \mathcal{A}}\left|q^{\prime}(u)\right|<1,
$$

then the scheme (5.4) admits a unique solution $\left(u_{j}^{n+1}\right)_{j \in \mathbb{Z}} \in L^{1}(\mathbb{Z})$.

Proof. The scheme (5.4) may be written

$$
u^{n+1}=\frac{\mu}{1+\mu} u^{n}+\frac{1}{1+\mu} T_{\mu}\left(u^{n+1}\right)+\frac{\mu}{1+\mu} k q\left(u^{n+1}\right) .
$$

Let us introduce the operator $T: L^{1}(\mathbb{Z}) \longrightarrow L^{1}(\mathbb{Z})$ defined by

$$
T(u)_{j}=\frac{1}{1+\mu} T_{\mu}(u)_{j}+\frac{\mu}{1+\mu} k q(u)_{j} \quad \forall j \in \mathbb{Z} .
$$

We have

$$
\begin{aligned}
\|T(u)-T(v)\|_{L^{1}(\mathbb{Z})} \leq & \frac{1}{1+\mu}\left\|T_{\mu}(u)-T_{\mu}(v)\right\|_{L^{1}(\mathbb{Z})} \\
& +\frac{\mu}{1+\mu} k \sup _{u \in \mathcal{A}}\left|q^{\prime}(u)\right|\|u-v\|_{L^{1}(\mathbb{Z})} .
\end{aligned}
$$

Using Lemma 5.1, we get

$$
\|T(u)-T(v)\|_{L^{1}(\mathbb{Z})} \leq C\|u-v\|_{L^{1}(\mathbb{Z})},
$$

where

$$
C=\frac{1}{1+\mu}+\frac{\mu}{1+\mu} k \sup _{u \in \mathcal{A}}\left|q^{\prime}(u)\right| .
$$

By condition (5.7), we have

$$
0<C<1 .
$$

Then $T$ is a contraction; therefore, there exists a unique solution of (5.4) in $L^{1}(\mathbb{Z})$.

Theorem 5.2. Under the subcharacteristic condition (3.4) the approximate solution $u_{h}$ constructed by the implicit scheme (5.4), converges in $L_{\mathrm{loc}}^{1}(\mathbb{R} \times] 0, T[)$ toward the entropy satisfying solution of (1.1)-(1.2), as $h$ tends to zero.

Proof. Using Proposition 5.2, the family of the approximate solution $\left(u_{h}\right)$ is bounded in $L^{\infty}(\mathbb{R} \times] 0, T[) \cap B V_{\text {loc }}(\mathbb{R} \times] 0, T[)$. Then by Helly's theorem, we can extract a subsequence still labeled $\left(u_{h}\right)$ which converges toward $u$ in $L_{\text {loc }}^{1}(\mathbb{R} \times] 0, T[)$. To show that $u$ satisfies the entropy condition (2.3), we proceed exactly as in [5].

Remark 5.1. We point out that the former result was proved without any CFL condition. 
5.3. The MUSCL semi-implicit relaxed scheme. To construct a second order accurate (in space) scheme using the Van-Leer method ([30]), we will need the initial boundary values inside cell $j$ :

$$
\begin{gathered}
\left(v^{n}+\sqrt{a} u^{n}\right)_{j+1 / 2}=\left(v^{n}+\sqrt{a} u^{n}\right)_{j}+1 / 2 h \delta_{j}^{+n}, \\
\left(v^{n}-\sqrt{a} u^{n}\right)_{j+1 / 2}=\left(v^{n}-\sqrt{a} u^{n}\right)_{j+1}-1 / 2 h \delta_{j+1}^{-n},
\end{gathered}
$$

with

$$
\delta_{j}^{ \pm}=\frac{1}{h}\left(v_{j+1} \pm \sqrt{a} u_{j+1}-v_{j} \pm \sqrt{a} u_{j}\right) \phi\left(\theta_{j}^{ \pm}\right)
$$

and

$$
\theta_{j}^{ \pm}=\frac{v_{j} \pm \sqrt{a} u_{j}-v_{j-1} \pm \sqrt{a} u_{j-1}}{v_{j+1} \pm \sqrt{a} u_{j+1}-v_{j} \pm \sqrt{a} u_{j}}
$$

where $\phi$ is a limiter function as defined by Sweby in [27] and satisfies

$$
0 \leq \frac{\phi(\theta)}{\theta} \leq 2, \quad 0 \leq \phi(\theta) \leq 2 .
$$

Then

$$
u_{j+1 / 2}^{n}=\frac{1}{2}\left(u_{j}^{n}+u_{j+1}^{n}\right)-\frac{1}{2 \sqrt{a}}\left(v_{j+1}^{n}-v_{j}^{n}\right)+\frac{h}{4 \sqrt{a}}\left(\delta_{j}^{+n}+\delta_{j+1}^{-n}\right),
$$

and

$$
v_{j+1 / 2}^{n}=\frac{1}{2}\left(v_{j}^{n}+v_{j+1}^{n}\right)-\frac{\sqrt{a}}{2}\left(u_{j+1}^{n}-u_{j}^{n}\right)+\frac{h}{4}\left(\delta_{j}^{+n}-\delta_{j+1}^{-n}\right) .
$$

Then the second order (in space) accurate semi-implicit relaxed scheme is given by

$$
\left\{\begin{array}{l}
v_{j}^{n}=f\left(u_{j}^{n}\right) \\
\begin{array}{r}
u_{j}^{n+1}=u_{j}^{n}-\frac{r}{2}\left(f\left(u_{j+1}^{n}\right)-f\left(u_{j-1}^{n}\right)\right)+\frac{\sqrt{a} r}{2}\left(u_{j+1}^{n}-2 u_{j}^{n}+u_{j-1}^{n}\right) \\
\quad+\frac{k}{4}\left(\delta_{j+1}^{-n}-\delta_{j}^{n}+\delta_{j-1}^{+n}-\delta_{j}^{+n}\right)+k q\left(u_{j}^{n+1}\right) .
\end{array}
\end{array}\right.
$$

Let

$$
C_{j}=\frac{1}{2} r\left(\sqrt{a}+\frac{f\left(u_{j+1}\right)-f\left(u_{j}\right)}{u_{j+1}-u_{j}}\right), \quad D_{j}=\frac{1}{2} r\left(\sqrt{a}-\frac{f\left(u_{j+1}\right)-f\left(u_{j}\right)}{u_{j+1}-u_{j}}\right) .
$$

By the subcharacteristic condition (3.4), we hav

$$
C_{j} \geq 0, \quad D_{j} \geq 0 .
$$

The scheme (5.11) may be written in the form

$$
u_{j}^{n+1}=\bar{u}_{j}^{n+1}+k q\left(u_{j}^{n+1}\right),
$$

with

$$
\begin{aligned}
\bar{u}_{j}^{n+1}= & u_{j}^{n}-C_{j-1}^{n}\left(u_{j}^{n}-u_{j-1}^{n}\right)+D_{j}^{n}\left(u_{j+1}^{n}-u_{j}^{n}\right) \\
& +\frac{k}{4}(1-r \sqrt{a})\left(\delta_{j+1}^{-n}-\delta_{j}^{-n}+\delta_{j-1}^{+n}-\delta_{j}^{+n}\right) .
\end{aligned}
$$

By using (5.9) and the definition of $C_{j}$ and $D_{j}$, we have

$$
\delta_{j}^{+}=\frac{2}{r h} C_{j} \phi_{j}^{+}\left(u_{j+1}-u_{j}\right), \quad \delta_{j}^{-}=-\frac{2}{r h} D_{j} \phi_{j}^{-}\left(u_{j+1}-u_{j}\right) .
$$


Dropping $n$ in the right-hand side, (5.13) gives

$$
\begin{aligned}
\bar{u}_{j}^{n+1}= & u_{j}-C_{j-1}\left(u_{j}-u_{j-1}\right)+D_{j}\left(u_{j+1}-u_{j}\right) \\
& -\frac{1}{2} \alpha D_{j+1} \phi_{j+1}^{-}\left(u_{j+2}-u_{j+1}\right)+\frac{1}{2} \alpha D_{j} \phi_{j}^{-}\left(u_{j+1}-u_{j}\right) \\
& +\frac{1}{2} \alpha C_{j-1} \phi_{j-1}^{+}\left(u_{j}-u_{j-1}\right)-\frac{1}{2} \alpha C_{j} \phi_{j}^{+}\left(u_{j+1}-u_{j}\right),
\end{aligned}
$$

where

$$
\alpha=(1-r \sqrt{a}) .
$$

Taking into account the equalities

$$
\theta_{j}^{+} C_{j}\left(u_{j+1}-u_{j}\right)=C_{j-1}\left(u_{j}-u_{j-1}\right), \quad \theta_{j}^{-} D_{j}\left(u_{j+1}-u_{j}\right)=D_{j-1}\left(u_{j}-u_{j-1}\right),
$$

this leads to

$$
\begin{aligned}
\bar{u}_{j}^{n+1} & =\left[1+\frac{1}{2} \alpha\left(\frac{\phi_{j}^{+}}{\theta_{j}^{+}}-\phi_{j-1}^{+}\right)\right] C_{j-1} u_{j-1} \\
& +\left(1-\left(1-\frac{1}{2} \alpha \phi_{j-1}^{+}+\frac{1}{2} \alpha \frac{\phi_{j}^{+}}{\theta_{j}^{+}}\right) C_{j-1}-D_{j-1}-\frac{1}{2} \alpha\left(\phi_{j}^{-}-\frac{\phi_{j+1}^{-}}{\theta_{j+1}^{-}}\right) D_{j}\right) u_{j} \\
& +\left(1-\frac{1}{2} \alpha\left(\frac{\phi_{j+1}^{-}}{\theta_{j+1}^{-}}-\phi_{j}^{-}\right)\right) D_{j} u_{j+1} .
\end{aligned}
$$

Now, we prove that the coefficients of $u_{j-1}, u_{j}$ and $u_{j+1}$ in (5.15) are positive.

Lemma 5.2. Under the subcharacteristic condition (3.4) and the CFL condition

$$
\nu=r \sqrt{a} \leq \frac{1}{2}
$$

the coefficients

$$
\begin{gathered}
A_{1, j}=\left[1+\frac{1}{2} \alpha\left(\frac{\phi_{j}^{+}}{\theta_{j}^{+}}-\phi_{j-1}^{+}\right)\right] C_{j-1}, \\
A_{2, j}=1-\left(1-\frac{1}{2} \alpha \phi_{j-1}^{+}+\frac{1}{2} \alpha \frac{\phi_{j}^{+}}{\theta_{j}^{+}}\right) C_{j-1}-D_{j-1}-\frac{1}{2} \alpha\left(\phi_{j}^{-}-\frac{\phi_{j+1}^{-}}{\theta_{j+1}^{-}}\right) D_{j}, \\
A_{3, j}=\left[1-\frac{1}{2} \alpha\left(\frac{\phi_{j+1}^{-}}{\theta_{j+1}^{-}}-\phi_{j}^{-}\right)\right] D_{j}
\end{gathered}
$$

are positive.

Proof. From (5.10) we have that

$$
\left|\frac{\phi_{j}^{+}}{\theta_{j}^{+}}-\phi_{j-1}^{+}\right| \leq 2 .
$$

Then

$$
1+\frac{1}{2} \alpha\left(\frac{\phi_{j}^{+}}{\theta_{j}^{+}}-\phi_{j-1}^{+}\right) \geq 1-\alpha=1-(1-\nu)=\nu \geq 0 ;
$$

$C_{j}$ being positive, then the coefficient $A_{1, j}$ is positive. By a proof similar to the former one, we get that $A_{3, j}$ is positive since $D_{j}$ is positive. 
Let us now prove that $A_{2, j}$ is positive.

$$
A_{2, j}=1+\frac{1}{2} \alpha \phi_{j-1}^{+} C_{j-1}-\left(C_{j-1}+D_{j-1}\right)-\frac{1}{2} \alpha\left[\frac{\phi_{j}^{+}}{\theta_{j}^{+}} C_{j-1}+\left(\phi_{j}^{-}-\frac{\phi_{j+1}^{-}}{\theta_{j+1}^{-}}\right) D_{j}\right] .
$$

The definition of $C_{j}$ and $D_{j}$ gives

$$
C_{j-1}+D_{j-1}=r \sqrt{a}=\nu \leq \frac{1}{2} .
$$

Hence,

$$
\begin{aligned}
A_{2, j} & \geq 1+\frac{1}{2} \alpha \phi_{j-1}^{+} C_{j-1}-\nu-\alpha\left(C_{j-1}+D_{j}\right) \\
& \geq 1+\frac{1}{2} \alpha \phi_{j-1}^{+} C_{j-1}-\nu-\alpha \nu-r \alpha \sqrt{a} \\
& \geq \frac{1}{2} \alpha \phi_{j-1}^{+} C_{j-1}+2 \nu^{2}-3 \nu+1 \\
& \geq \frac{1}{2} \alpha \phi_{j-1}^{+} C_{j-1} \quad \text { since } \nu \leq \frac{1}{2} \\
& \geq 0
\end{aligned}
$$

Proposition 5.4. Under the subcharacteristic condition (3.4) and the CFL condition (5.16), the scheme (5.11) is $L^{\infty} D$. That is

$$
\left\|u^{n+1}\right\|_{L^{\infty}(\mathbb{Z})} \leq\left\|u^{n}\right\|_{L^{\infty}(\mathbb{Z})} .
$$

Proof. The scheme (5.11) may be written

$$
u_{j}^{n+1}=\bar{u}_{j}^{n+1}+k q\left(u_{j}^{n+1}\right),
$$

with

$$
\begin{aligned}
\bar{u}_{j}^{n+1}= & {\left[1+\frac{1}{2} \alpha\left(\frac{\phi_{j}^{+}}{\theta_{j}^{+}}-\phi_{j-1}^{+}\right)\right] C_{j-1} u_{j-1} } \\
& \left.+\left(1-\frac{1}{2} \alpha \phi_{j-1}^{+}+\frac{1}{2} \alpha \frac{\phi_{j}^{+}}{\theta_{j}^{+}}\right) C_{j-1}-D_{j-1}-\frac{1}{2} \alpha\left(\phi_{j}^{-}-\frac{\phi_{j+1}^{-}}{\theta_{j+1}^{-}}\right) D_{j}\right] u_{j} \\
& +\left[1-\frac{1}{2} \alpha\left(\frac{\phi_{j+1}^{-}}{\theta_{j+1}^{-}}-\phi_{j}^{-}\right)\right] D_{j} u_{j+1} .
\end{aligned}
$$

Let us first prove that

$$
\left\|\bar{u}^{n+1}\right\|_{L^{\infty}(\mathbb{Z})} \leq\left\|u^{n}\right\|_{L^{\infty}(\mathbb{Z})}
$$

indeed,

$$
\bar{u}_{j}^{n+1}=A_{1, j} u_{j-1}^{n}+A_{2, j} u_{j}^{n}+A_{3, j} u_{j+1}^{n} .
$$

By Lemma 5.2, $A_{1, j}, A_{2, j}$ and $A_{3, j}$ are positive under the CFL condition (5.16). Hence

$$
\begin{aligned}
\left|\bar{u}_{j}^{n+1}\right| & \leq A_{1, j}\left|u_{j-1}^{n}\right|+A_{2, j}\left|u_{j}^{n}\right|+A_{3, j}\left|u_{j+1}^{n}\right| \\
& \leq A_{1, j}\left\|u^{n}\right\|_{L^{\infty}(\mathbb{Z})}+A_{2, j}\left\|u^{n}\right\|_{L^{\infty}(\mathbb{Z})}+A_{3, j}\left\|u^{n}\right\|_{L^{\infty}(\mathbb{Z})} .
\end{aligned}
$$

After simplification we get

$$
\left|\bar{u}_{j}^{n+1}\right| \leq\left\|u^{n}\right\|_{L^{\infty}(\mathbb{Z})} \quad \forall j \in \mathbb{Z},
$$


since

$$
A_{1, j}+A_{2, j}+A_{3, j}=1 \text {. }
$$

Then

$$
\left\|\bar{u}^{n+1}\right\|_{L^{\infty}(\mathbb{Z})} \leq\left\|u^{n}\right\|_{L^{\infty}(\mathbb{Z})} \cdot
$$

Using the mean value formula in (5.18) and then as in the proof of Proposition 5.1, we obtain

$$
\left\|u^{n+1}\right\|_{L^{\infty}(\mathbb{Z})} \leq\left\|u^{n}\right\|_{L^{\infty}(\mathbb{Z})} .
$$

By a similar proof to that of Proposition 5.4, we may prove

Proposition 5.5. Under the subcharacteristic condition (3.4) and the CFL condition (5.16), the scheme (5.20) satisfies

i) $T V\left(u^{n+1}\right) \leq T V\left(u^{n}\right)$;

ii) $\sum_{|j| \leq J}\left|u_{j}^{n+1}-u_{j}^{n}\right| \leq C_{1} T V\left(u^{n}\right)+C_{2}\left\|u^{n+1}\right\|_{L^{\infty}(\mathbb{Z})}$.

Using Propositions 5.4 and 5.5, we get that the family of the approximate solutions $\left(u_{h}\right)$ is bounded in $L^{\infty}(\mathbb{R} \times] 0, T[) \cap B V_{\text {loc }}(\mathbb{R} \times] 0, T[)$ and then we are able to prove the following result.

Theorem 5.3. Under the subcharacteristic condition (3.4) and the CFL condition (5.16), there exists a subsequence from the approximate family $\left(u_{h}\right)$, which converges in $L_{\mathrm{loc}}^{1}(\mathbb{R} \times] 0, T[)$ toward a weak solution of $(1.1)-(1.2)$ as $h$ tends to 0.

Remark 5.2. Time discretization may be achieved using the Runge-Kutta method as in [6] and [13].

\section{Extension to THE TWO DIMENSIONAL CASE}

We consider now the two dimensional Cauchy problem:

$$
\left.u_{t}+f(u)_{x}+g(u)_{y}=q(u) ; \quad(x, y, t) \in \mathbb{R}^{2} \times\right] 0, T[; T>0
$$

and

$$
u(x, y, 0)=u_{0}(x, y) ; \quad(x, y) \in \mathbb{R}^{2} .
$$

In this case, we assume that

$$
u_{0} \in B V\left(\mathbb{R}^{2}\right) ; \quad f, g \in \mathcal{C}^{1}(\mathbb{R})
$$

and

$$
q \in \mathcal{C}^{1}(\mathbb{R}), \quad q(0)=0, \quad q^{\prime} \leq 0 .
$$

The existence and uniqueness of the solution of (6.1)-(6.2) is established by Kruzkov in [18].

The associated relaxation system with (6.1) is

$$
\left\{\begin{array}{l}
u_{t}+v_{x}+w_{y}=q(u), \\
v_{t}+a u_{x}=-\frac{1}{\epsilon}(v-f(u)), \\
w_{t}+b u_{y}=-\frac{1}{\epsilon}(w-g(u)) .
\end{array}\right.
$$


As in the one dimensional case, the system (6.3) is dissipative under the following subcharacteristic condition:

$$
\frac{\left(f^{\prime}(u)\right)^{2}}{a}+\frac{\left(g^{\prime}(u)\right)^{2}}{b} \leq 1 \quad \forall u .
$$

Let

$$
r=\frac{\Delta t}{\Delta x}, \quad s=\frac{\Delta t}{\Delta y} .
$$

Using the same method as in Section 5, the relaxed scheme for (6.1)-(6.2) is

$$
\left\{\begin{array}{l}
v_{i, j}^{n}=f\left(u_{i, j}^{n}\right) \\
w_{i, j}^{n}=g\left(u_{i, j}^{n}\right) \\
u_{i, j}^{n+1}=u_{i, j}^{n}-\frac{r}{2}\left(f\left(u_{i+1, j}^{n}\right)-f\left(u_{i-1, j}^{n}\right)\right)+\frac{\sqrt{a} r}{2}\left(u_{i+1, j}^{n}-2 u_{i, j}^{n}+u_{i-1, j}^{n}\right) \\
\quad-\frac{s}{2}\left(g\left(u_{i, j+1}^{n}\right)-g\left(u_{i, j-1}^{n}\right)\right)+\frac{\sqrt{b} s}{2}\left(u_{1, j+1}^{n}-2 u_{i, j}^{n}+u_{i, j-1}^{n}\right)+\Delta t q\left(u_{i, j}^{n+1}\right)
\end{array}\right.
$$

with

$$
u_{i, j}^{0}=\frac{1}{\Delta x \Delta y} \int_{(j-1 / 2) \Delta x}^{(j+1 / 2) \Delta x} \int_{(j-1 / 2) \Delta y}^{(j+1 / 2) \Delta y} u_{0}(x, y) d x d y .
$$

A solution of (6.1)-(6.2) is approximated by a function $u_{h}$ defined on $\left.\mathbb{R}^{2} \times\right] 0, T$ [ by

$$
\begin{aligned}
\left.u_{h}(x, y, t)=u_{i, j}^{n} \quad \text { for } \quad(x, y, t) \in\right] & (i-1 / 2) \Delta x,(i+1 / 2) \Delta x[ \\
& \times](j-1 / 2) \Delta y,(j+1 / 2) \Delta y[\times[n \Delta t,(n+1) \Delta t[
\end{aligned}
$$

with

$$
(i, j) \in \mathbb{Z}^{2} \text { and } n \leq N=E(T / k)+1 .
$$

Proposition 6.1. Under the subcharacteristic condition (6.4) and the CFL condition

$$
\nu=r \sqrt{a}+s \sqrt{b} \leq 1
$$

the scheme (6.5) satisfies

i) $\left\|u^{n+1}\right\|_{L^{\infty}(\mathbb{Z} \times \mathbb{Z})} \leq\left\|u^{n}\right\|_{L^{\infty}(\mathbb{Z} \times \mathbb{Z})}$,

ii) $T V\left(u^{n+1}\right) \leq T V\left(u^{n}\right)$,

iii) $\sum_{|i| \leq I,|j| \leq J}\left|u_{i, j}^{n+1}-u_{i, j}^{n}\right| \leq C_{1} T V\left(u^{n}\right)+C_{2}\left\|u^{n+1}\right\|_{L^{\infty}(\mathbb{Z} \times \mathbb{Z})}$,

where

$$
\text { || } u^{n} \|_{L^{\infty}(\mathbb{Z} \times \mathbb{Z})}=\sup _{i, j \in \mathbb{Z}}\left|u_{i, j}^{n}\right|
$$

and

$$
T V\left(u^{n}\right)=\sum_{i, j \in \mathbb{Z}}\left|u_{i+1, j}^{n}-u_{i, j}^{n}\right| \Delta y+\sum_{i, j \in \mathbb{Z}}\left|u_{i, j+1}^{n}-u_{i, j}^{n}\right| \Delta x .
$$

The proof is similar to those of Propositions 5.1-5.5.

As in subsection 5.1, using Proposition 6.1 we prove the following theorem. 
Theorem 6.1. Under the subcharacteristic (6.4) and the CFL condition (6.7), the approximate solution $u_{h}$ given by the relaxed scheme (6.5) converges toward the entropy solution of (6.1)-(6.2).

Remark 6.1. Second order accurate schemes may be constructed as in subsection 5.3 ; however these schemes will not be TVD (see [12]).

\section{Conclusion}

We presented an analysis of a class of relaxation schemes for hyperbolic conservation laws including stiff source terms. This method was introduced for the first time by Jin and Xin in [16] for the approximation of hyperbolic conservation laws without source terms. The construction of these schemes is based on the approximation of an associated linear hyperbolic system with a stiff source term depending on a small parameter $\epsilon$ (relaxation time). This method allows us to avoid the use of a Riemann solver.

With an adequate and realistic hypothesis, $\left(q^{\prime}(u) \leq 0\right)$ on the source term $q(u)$, that is with a dissipative (in some sense) source $q(u)$, we proposed semi-implicit and fully implicit numerical schemes possessing good properties, such as monotony, TVD character, etc., exactly as in the case of conservation laws without a source term $(q=0)$.

Under reasonable CFL conditions, we established the convergence of the approximate solution toward a weak solution or to the entropy satisfying solution (in some cases).

An extension of this analysis to the multidimensional case is given at the end of this paper.

We think that a direct study (stability, bound error, convergence, etc.) of the relaxing schemes (4.1), which depend on the relaxation rate $\epsilon$, will be of interest, especially as concerns the link between this small parameter $\epsilon$ and the discretization steps $\Delta t$ and $\Delta x$.

\section{REFERENCES}

[1] D. Aregba-Driollet and R. Natalini, Convergence of relaxation schemes for conservation laws, Appl. Anal. 61 (1996), 163-193. CMP 98:13

[2] A. C. Berkenbosch, E. F. Kaasschieter, and J. H. M. Ten Thije Boonkkamp, The numerical wave speed for one-dimensional scalar conservation laws with source terms. Preprint, Eindhoven University of Technology, (1994).

[3] A. Bermudez and M. E. Vazquez, Upwind methods for hyperbolic conservation laws with source terms, Comput. \& Fluids, 23 (1994), pp. 1049-1071. MR 95i:76065

[4] A. Chalabi, Stable upwind schemes for hyperbolic conservation laws with source terms, IMA J. Numer. Anal., 12 (1992), pp. 217-242. MR 93c:65108

[5] A. Chalabi, An error bound for the polygonal approximation of conservation laws with source terms, Comput. \& Math. Appl., 32 (1996), pp. 59-63. MR 97i:65143

[6] A. Chalabi, On convergence of numerical schemes for hyperbolic conservation laws with stiff source terms, Math. Comp., 66 (1997), pp. 527-545. MR 97g:65178

[7] G. Q. Chen, C. D. Levermore and T. P. Liu, Hyperbolic conservation laws with stiff relaxation terms and entropy, Comm. Pure Appl. Math., 47 (1994), pp. 787-830. MR 95h:35133

[8] P. Colella, A. Majda and V. Roytburd, Theoretical and numerical structure for reacting shock waves, SIAM J. Sci. Stat. Comput., 7 (1986), pp. 1059-1080. MR 87i:76037

[9] J. F. Collet and M. Rascle, Convergence of the relaxation approximation to a scalar nonlinear hyperbolic equation arising in chromatography, Z. Angew. Math. Phys. 47 (1996), 400-409. MR 97d:35132 
[10] M. G. Crandall and A. Majda, Monotone difference approximations for scalar conservation conservation laws, Math. Comp., 34 (1980), pp. 1-21. MR 81b:65079

[11] B. Engquist and B. Sjogreen, Robust difference approximations of stiff inviscid detonation waves, CAM report 91-03, UCLA, Los Angeles, CA, 1991.

[12] J. B. Goodman and R. Leveque, On the accuracy of stable schemes for 2D scalar conservation laws, Math. Comp., 45 (1985), pp. 15-21. MR 86f:65149

[13] S. Jin, Runge-Kutta methods for hyperbolic conservation laws with stiff relaxation terms, J. Comput. Phys., 122 (1995), pp. 51-67. MR 96g:65084

[14] S. Jin, A convex entropy for a hyperbolic system with relaxation, J. Diff. Equations, 127 (1996), pp. 97-109. MR 97c:35130

[15] S. Jin and C. D. Levermore, Numerical schemes for hyperbolic conservation laws with stiff relaxation terms, J. Comput. Phys., 126 (1996), pp. 449-467. MR 97g:65173

[16] S. Jin and Z. Xin, The relaxation schemes for systems of conservation laws in arbitrary space dimensions, Comm. Pure Appl. Math., 48 (1995), pp. 235-276. MR 96c:65134

[17] P. Klingenstein, Hyperbolic conservation laws with source terms: Errors of the shock location, PhD thesis, Suiss Federal Institute of Tecnology, Zürich (1997).

[18] S. N. Kruzkov, First order quasi-linear equations in several independent variables, Math. USSR-Sb., 81 (1970), pp. 228-255. MR 42:2159

[19] J. LeVeque and H. C. Yee, A study of numerical methods for hyperbolic conservation laws with stiff source terms, J. Comput. Phys., 86 (1990), pp. 187-210. MR 90k:76009

[20] T. P. Liu, Hyperbolic conservation laws with relaxation, Comm. Math. Phys., 108 (1987), pp. 153-175. MR 88f:35092

[21] A. Majda, A qualitative model for dynamic combustion, SIAM J. Appl. Math., 40 (1981), pp. 70-93. MR 82j:35096

[22] R. Natalini, Convergence to equilibrium for the relaxation approximations of conservation laws, Comm. Pure Appl. Math. 49 (1996), pp. 795-823. MR 97c:35131

[23] S. Osher, Riemann solvers, the entropy condition, and difference approximations, SIAM J. Numer. Anal., 21 (1984), pp. 217-235. MR 86d:65119

[24] R. B. Pember, Numerical methods for hyperbolic conservation laws with stiff relaxation II. Higher-order Godunov methods, SIAM J. Sci. Comput., 14 (1993), pp. 824-859. MR 95f: 65190

[25] H. J. Schroll, A. Tveito and R. Winther, An $L_{1}$ error bound for semi-implicit difference scheme applied to a stiff system of conservation laws, SIAM J. Numer. Anal. 34 (1997), 1152-1166. MR 98g:65078

[26] H. J. Schroll and R. Winther, Finite difference schemes for conservation laws with source terms, IMA J. Numer. Anal., 16 (1996), pp. 201-215. MR 97g:65177

[27] P. R. Sweby, High resolution schemes using flux limiters for hyperbolic conservation laws, SIAM J. Numer. Anal. 21 (1984), pp. 995-1011. MR 85m:65085

[28] E. Tadmor, Numerical viscosity and the entropy condition for conservative difference schemes, Math. Comp. 43 (1984), pp. 369-381. MR 86g:65163

[29] T. Tang and Z. H. Teng, Error bounds for fractional step methods for conservation laws with source terms, SIAM J. Numer. Anal., 32 (1995), pp. 110-127. MR 95m:65155

[30] B. Van Leer, Towards the ultimate conservative difference schemes $V$. A second order sequel to Godunov's method, J. Comput. Phys., 32 (1979), pp. 101-136. CMP 98:05

[31] J. P. Vila, Convergence and error estimates in finite volume schemes for multidimensional scalar conservation laws, Preprint, Nice University (1995).

[32] G. B. Whitham, Linear and nonlinear waves, J. Wiley (1974). MR 58:3905

CNRS, Umr Mip 5640 - UfR Mig Universite P. Sabatier, Route de Narbonne 31062 TOUlOuse CEDEX France

E-mail address: chalabi@mip.ups-tlse.fr 